\title{
WEIGHTED COMPOSITION OPERATORS ON ORLICZ_SOBOLEV SPACES
}

\author{
SUBHASH C. ARORA ${ }^{\varpi}$, GOPAL DATT and SATISH VERMA
}

(Received 29 April 2005; revised 22 July 2006)

Communicated by A. J. Pryde

\begin{abstract}
For an open subse: $\Omega$ of the Euclidean space $\mathbf{R}^{n}$, a measurable non-singular transformation $T: \Omega \rightarrow \Omega$ and a real-valued measurable function $u$ on $\mathbf{R}^{n}$, we study the weighted composition operator $u C_{T}: f \mapsto u \cdot(f \circ T)$ on the Orlicz-Sobolev space $W^{1 . \psi}(\Omega)$ consisting of those functions of the Orlicz space $L^{\psi}(\Omega)$ whose distributional derivatives of the first order belong to $L^{\varphi}(\Omega)$. We also discuss a sufficient condition under which $\iota C_{T}$ is compact.
\end{abstract}

2000 Mathematics subject classification: primary 47B33. 47B38; secondary 46E30, 46E35.

\section{Introduction}

Let $\varphi:[0, \infty) \rightarrow[0, \infty)$ be a Young function $([1,6])$. Thus $\varphi$ is a continuous, convex, strictly increasing function satisfying $\varphi(0)=0, \lim _{t \rightarrow \infty} \varphi(t)=\infty$, $\lim _{t \rightarrow 0+} \varphi(t) / t=0$ and $\lim _{t \rightarrow+\infty} \varphi(t) / t=+\infty$. We say that $\varphi$ satisfies the $\Delta_{2^{-}}$ condition if there exist constants $k>0, t_{0} \geq 0$ such that $\varphi(2 t) \leq k \varphi(t)$ for all $t \geq t_{0}$. Associated with $\varphi$, we have the complementary Young function $\psi:[0, \infty) \rightarrow[0, \infty)$ defined by $\psi(s)=\sup \{s t-\varphi(t): t \geq 0\}$.

Let $(\Omega, \mathscr{A}, \mu)$ be a measure space where $\Omega$ is an open subset of the Euclidean space $\mathbf{R}^{n}$ and $\mathscr{A}$ be the $\sigma$-algebra of Lebesgue measurable subsets of $\Omega$ and $\mu$ be the Lebesgue measure. The Orlicz space $L^{\varphi}(\Omega)$ is defined as the set of all (equivalence classes of real-valued measurable functions $f$ on $\Omega$ such that $\|f\|_{\varphi}<\infty$, where $\|\cdot\|_{\varphi}$ denotes the Luxemberg norm defined by

$$
\|f\|_{\varphi}=\inf \left\{k>0: \int_{\Omega} \varphi\left(\frac{|f|}{k}\right) d \mu \leq 1\right\} .
$$

(C) 2007 Australian Mathematical Society $1446-7887 / 07 \$ A 2.00+0.00$ 
$L^{\varphi}(\Omega)$ is a Banach space with respect to the above norm.

The Orlicz-Sobolev space $W^{1 . \varphi}(\Omega)$ is defined as the set of all real-valued functions $f$ in $L^{\varphi}(\Omega)$ whose weak partial derivatives $\partial f / \partial x_{i}$ (in the distributional sense) belong to $L^{\varphi}(\Omega), i=1,2, \ldots, n$. It is a Banach space with respect to the norm:

$$
\|f\|_{1, \varphi}=\|f\|_{\varphi}+\sum_{i=1}^{n}\left\|\frac{\partial f}{\partial x_{i}}\right\|_{\varphi}
$$

On the $\sigma$-finite measure space $(\Omega, \mathscr{A}, \mu)$, let $T: \Omega \rightarrow \Omega$ be a measurable (that is $T^{-1}(A) \in \mathscr{A}$ for every $A \in \mathscr{A}$ ) non-singular transformation (that is, $\mu\left(T^{-1}(A)\right)=0$ whenever $\mu(A)=0)$. Let the function $f_{T}=d\left(\mu \circ T^{-1}\right) / d \mu$ be the Radon-Nikodym derivative. Suppose $u$ is a real-valued measurable function defined on $\mathbf{R}^{n}$. Then $T$ induces a well-defined weighted composition linear transformation $u C_{T}$ on $W^{1, \varphi}(\Omega)$ defined by

$$
\left(u C_{T} f\right)(x)=u(x) f(T(x)), \quad x \in \Omega, f \in W^{1 . \varphi}(\Omega) .
$$

If $u C_{T}$ maps $W^{1 . \varphi}(\Omega)$ into itself and is bounded then we call $u C_{T}$ a weighted composition operator on $W^{1 . \varphi}(\Omega)$ induced by $T$ with weight $u$. If $u \equiv 1$ then $C_{T}$ is called a composition operator induced by $T$.

Our present study of weighted composition operators on the Orlicz-Sobolev space $W^{1 . \varphi}(\Omega)$ is motivated by the work of Kamowitz and Wortman ([4]). Other similar references include ([2,3,5] and [7]). In Section 2 we define the composition operator on $W^{1 . \varphi}(\Omega)$ and Section 3 is devoted to the study of the compact weighted composition operator on $W^{1 . \varphi}(\Omega)$.

\section{Composition operator on $W^{1 . \varphi}(\Omega)$}

LEMMA 2.1. Let $f_{T}, \partial T_{k} / \partial x_{i} \in L^{\infty}(\mu)$ with $\left\|\partial T_{k} / \partial x_{i}\right\|_{\infty} \leq M, M>0$ for $i, k=1,2, \ldots, n$, where $T=\left(T_{1}, T_{2}, \ldots, T_{n}\right)$ and $\partial T_{k} / \partial x_{i}$ denotes the first order partial derivative (in the classical sense). Then for each $f$ in $W^{1 . \varphi}(\Omega)$ we have $f \circ T \in L^{\varphi}(\Omega)$ and if the Young function $\varphi$ satisfies the $\Delta_{2}$-condition then the first order distributional derivatives of $(f \circ T)$, given by

$$
\frac{\partial}{\partial x_{i}}(f \circ T)=\sum_{k=1}^{n}\left(\frac{\partial f}{\partial x_{k}} \circ T\right) \frac{\partial T_{k}}{\partial x_{i}}
$$

for $i=1,2, \ldots, n$, are in $L^{\varphi}(\Omega)$. 
PROOF. For $f$ in $W^{1, \varphi}(\Omega)$, we have

$$
\begin{aligned}
\|f \circ T\|_{\varphi} & =\inf \left\{k>0: \int_{\Omega} \varphi\left(\frac{1}{k}|f \circ T|\right) d \mu \leq 1\right\} \\
& \leq \inf \left\{k>0: \int_{\Omega} \varphi\left(\frac{|f|}{k}\right) f_{T} d \mu \leq 1\right\} \\
& \leq \inf \left\{k>0:\left\|f_{T}\right\|_{\infty} \int_{\Omega} \varphi\left(\frac{|f|}{k}\right) d \mu \leq 1\right\} \\
& \leq \inf \left\{k>0: \int_{\Omega} \varphi\left(\frac{|f|}{k}\right) d \mu \leq 1\right\}=\|f\|_{\varphi}
\end{aligned}
$$

provided $\left\|f_{T}\right\|_{\infty} \leq 1$. Therefore in this case $f \circ T \in L^{\varphi}(\Omega)$.

Now we assume that $1<\left\|f_{r}\right\|_{\infty}<\infty$. Then for $f \neq 0$ we have

$$
\begin{aligned}
\int_{\Omega} \varphi\left(\frac{|f \circ T|}{\left\|f_{T}\right\|_{\infty}\|f\|_{\varphi}}\right) d \mu & \leq \int_{\Omega} \varphi\left(\frac{|f|}{\left\|f_{T}\right\|_{\infty}\|f\|_{\varphi}}\right) f_{T} d \mu \\
& \leq \int_{\Omega} \frac{1}{\left\|f_{T}\right\|_{\infty}} \varphi\left(\frac{|f|}{\|f\|_{\varphi}}\right)\left\|f_{T}\right\|_{\infty} d \mu \leq 1 .
\end{aligned}
$$

Thus $\|f \circ T\|_{\varphi} \leq\left\|f_{T}\right\|_{\infty}\|f\|_{\varphi}$ and hence $f \circ T \in L^{\varphi}(\Omega)$.

By the same arguments as were used to show that $\partial f / \partial x_{k}$ is in $L^{\varphi}(\Omega)$, it follows that $\left(\partial f / \partial x_{k}\right) \circ T \in L^{\varphi}(\Omega)$, for each $k=1,2, \ldots, n$. Also $\partial T_{k} / \partial x_{i} \in L^{\infty}(\mu)$, therefore

$$
\left(\frac{\partial f}{\partial x_{k}} \circ T\right) \frac{\partial T_{k}}{\partial x_{i}} \in L^{\varphi}(\Omega) \quad \text { for each } i, k=1,2, \ldots, n .
$$

Hence, by the triangle inequality, it follows that the function on the right hand side of (2.1) belongs to $L^{\varphi}(\Omega)$ for each $i=1,2, \ldots, n$.

Now $f \in W^{1 . \varphi}(\Omega)$ and $\varphi$ satisfies the $\Delta_{2}$-condition, so there exists (by [1, Theorem 8.28(d) Page 247]) a sequence $\left\langle f_{m}\right\rangle$ in $C^{\infty}(\Omega) \cap W^{1 . \varphi}(\Omega)$ such that $f_{m} \rightarrow f$ in $W^{1 . \varphi}(\Omega)$. Hence $f_{m} \rightarrow f$ and for $i=1,2, \ldots, n$ we have

$$
\frac{\partial f_{m}}{\partial x_{i}} \rightarrow \frac{\partial f}{\partial x_{i}} \quad \text { in } L^{\varphi}(\Omega)
$$

Let $g \in \mathscr{D}(\Omega)$ (the space of all infinitely differentiable real-valued functions with compact support in $\Omega$ ). Then, by the ordinary chain rule for the smooth function $f_{m}$, we have

$$
\int_{\Omega}\left(f_{m} \circ T\right) \frac{\partial g}{\partial x_{i}} d \mu=-\int_{\Omega} \frac{\partial}{\partial x_{i}}\left(f_{m} \circ T\right) g d \mu=\int_{\Omega} \sum_{k=1}^{n}\left(\frac{\partial f_{m}}{\partial x_{k}} \circ T\right) \frac{\partial T_{k}}{\partial x_{i}} g d \mu
$$

for each $i=1,2, \ldots, n$. 
Now $g \in \mathscr{D}(\Omega)$ implies that $g, \partial g / \partial x_{i} \in L^{\psi}(\Omega)$ for each $i$, where $\psi$ is the complementary Young function to $\varphi$. Therefore, by using the generalized version of Hölder's inequality in Orlicz spaces, we have

$$
\begin{aligned}
\int_{\Omega}\left|f_{m} \circ T-f \circ T\right|\left|\frac{\partial g}{\partial x_{i}}\right| d \mu & \leq 2\left\|\left(f_{m}-f\right) \circ T\right\|_{\varphi}\left\|\frac{\partial g}{\partial x_{i}}\right\|_{\psi} \\
& \leq 2\left\|f_{r}\right\|_{\infty}\left\|f_{m}-f\right\|_{\varphi}\left\|\frac{\partial g}{\partial x_{i}}\right\|_{\psi} \rightarrow 0 .
\end{aligned}
$$

Therefore

$$
\int_{\Omega}\left(f_{m} \circ T\right) \frac{\partial g}{\partial x_{i}} d \mu \quad \rightarrow \quad \int_{\Omega}(f \circ T) \frac{\partial g}{\partial x_{i}} d \mu
$$

as $m \rightarrow \infty$ for $i=1,2, \ldots n$.

By similar arguments, using $\partial f_{m} / \partial x_{k} \rightarrow \partial f / \partial x_{k}$ in $L^{\varphi}(\Omega)$ and $\partial T_{k} / \partial x_{i}$ in $L^{\infty}(\mu)$, we deduce that

$$
\sum_{k=1}^{n}\left(\frac{\partial f_{m}}{\partial x_{k}} \circ T\right) \frac{\partial T_{k}}{\partial x_{i}} \rightarrow \sum_{k=1}^{n}\left(\frac{\partial f}{\partial x_{k}} \circ T\right) \frac{\partial T_{k}}{\partial x_{i}}
$$

in $L^{\varphi}(\Omega)$, for each $i=1,2 \ldots n$, and so by Hölder's inequality again we obtain as $m \rightarrow \infty$,

$$
\int_{\Omega} \sum_{k=1}^{n}\left(\frac{\partial f_{m}}{\partial x_{k}} \circ T\right) \frac{\partial T_{k}}{\partial x_{i}} g d \mu \rightarrow \int_{\Omega} \sum_{k=1}^{n}\left(\frac{\partial f}{\partial x_{k}} \circ T\right) \frac{\partial T_{k}}{\partial x_{i}} g d \mu .
$$

Hence, by taking limits on both the sides of (2.2) as $m \rightarrow \infty$, we obtain

$$
\int_{\Omega}(f \circ T) \frac{\partial g}{\partial x_{i}} d \mu=-\int_{\Omega} \sum_{k=1}^{n}\left(\frac{\partial f}{\partial x_{k}} \circ T\right) \frac{\partial T_{k}}{\partial x_{i}} g d \mu .
$$

Therefore

$$
-\int_{\Omega} \frac{\partial}{\partial x_{i}}(f \circ T) g d \mu=-\int_{\Omega} \sum_{k=1}^{n}\left(\frac{\partial f}{\partial x_{k}} \circ T\right) \frac{\partial T_{k}}{\partial x_{i}} g d \mu .
$$

for all $i=1.2, \ldots, n$.

As $g$ was chosen arbitrarily, Equation (2.1) follows for $i=1,2, \ldots n$.

THEOREM 2.2. Let $\Omega \subset \mathbf{R}^{n}$ be an open set and $T: \Omega \rightarrow \Omega$ a measurable non-singular transformation with $f_{T}=d\left(\mu \circ T^{-1}\right) / d \mu,\left(\partial T_{k} / \partial x_{i}\right)$ in $L^{\infty}(\mu)$ and $\left\|\partial T_{k} / \partial x_{i}\right\|_{\infty} \leq M, M>0$, for $i, k=1,2, \ldots n$, where $T=\left(T_{1}, T_{2}, \ldots T_{n}\right)$ and $\partial T_{k} / \partial x_{i}$ denotes the first order partial derivative in the classical sense. Then for a Young function $\varphi$ satisfying the $\Delta_{2}$-condition, the mapping $C_{T}$ defined by $C_{T}(f)=f \circ T$ is a composition operator on the Orlicz-Sobolev space $W^{1 . \varphi}(\Omega)$. 
PROOF. By the Lemma 2.1, we have $f \circ T \in W^{1, \varphi}(\Omega)$ and

$$
\begin{aligned}
\|f \circ T\|_{1, \varphi} & =\|f \circ T\|_{\varphi}+\sum_{i=1}^{n}\left\|\frac{\partial}{\partial x_{i}}(f \circ T)\right\|_{\varphi} \\
& =\|f \circ T\|_{\varphi}+\sum_{i=1}^{n}\left\|\sum_{k=1}^{n}\left(\frac{\partial f}{\partial x_{k}} \circ T\right) \frac{\partial T_{k}}{\partial x_{i}}\right\|_{\varphi} \\
& \leq\left\|f_{T}\right\|_{\infty}\|f\|_{\varphi}+\sum_{i=1}^{n} \sum_{k=1}^{n}\left\|f_{T}\right\|_{\infty}\left\|\frac{\partial f}{\partial x_{k}}\right\|_{\varphi}\left\|\frac{\partial T_{k}}{\partial x_{i}}\right\|_{\infty} \\
& \leq\left\|f_{T}\right\|_{\infty}\|f\|_{\varphi}+\left\|f_{T}\right\|_{\infty} M n \sum_{k=1}^{n}\left\|\frac{\partial f}{\partial x_{k}}\right\|_{\varphi} \leq\left\|f_{T}\right\|_{\infty}(1+n M)\|f\|_{1 . \varphi} .
\end{aligned}
$$

The result follows.

\section{Compact weighted composition operator on $W^{1, \varphi}(\Omega)$}

Suppose $u$ is a real-valued measurable function on $\mathbf{R}^{n}$ and $T: \Omega \rightarrow \Omega$ is a measurable non-singular transformation and $(\Omega, \mathscr{A}, \mu)$ is the $\sigma$-finite measure space, where $\Omega$ an open subset of $\mathbf{R}^{n}$. On the same lines as in Lemma 2.1 , we have the following.

LEMMA 3.1. If all the conditions stated in Theorem 2.2 are satisfied and, in addition, $u \in L^{\infty}(\mu)$ is such that the first order classical partial derivatives $\partial u / \partial x_{i}$ satisfy $\left\|\partial u / \partial x_{i}\right\|_{\infty} \leq M_{1}, M_{1}>0$, for $i=1,2, \ldots, n$, then the mapping $u C_{T}$ defined by

$$
\left(u C_{T}\right) f=u \cdot(f \circ T)
$$

is a weighted composition operator on the Orlicz-Sobolev space $W^{1: \varphi}(\Omega)$.

PROOF. By the same arguments as in Lemma 2.1, we-find

$$
\frac{\partial}{\partial x_{i}}(u \cdot(f \circ T))=\frac{\partial u}{\partial x_{i}}(f \circ T)+u \sum_{k=1}^{n}\left(\frac{\partial f}{\partial x_{k}} \circ T\right) \frac{\partial T_{k}}{\partial x_{i}} \text { for } i=1,2, \ldots, n \text {. }
$$

Moreover

$$
\begin{gathered}
\|u \cdot(f \circ T)\|_{\varphi} \leq\|u\|_{\infty}\left\|f_{T}\right\|_{\infty}\|f\|_{\varphi}, \\
\left\|\frac{\partial u}{\partial x_{i}}(f \circ T)\right\|_{\varphi} \leq M_{1}\left\|f_{T}\right\|_{\infty}\|f\|_{\varphi} \text { and } \\
\left\|u \sum_{k=1}^{n}\left(\frac{\partial f}{\partial x_{k}} \circ T\right) \frac{\partial T_{k}}{\partial x_{i}}\right\|_{\varphi} \leq\|u\|_{\infty} M\left\|f_{T}\right\|_{\infty} \sum_{k=1}^{n}\left\|\frac{\partial f}{\partial x_{k}}\right\|_{\varphi} .
\end{gathered}
$$


Hence it follows that

$$
\begin{aligned}
& \left\|\left(u C_{T}\right) f\right\|_{1, \varphi} \\
& \quad=\|u \cdot(f \circ T)\|_{1, \varphi}=\|u \cdot(f \circ T)\|_{\varphi}+\sum_{i=1}^{n}\left\|\frac{\partial}{\partial x_{i}}(u \cdot(f \circ T))\right\|_{\varphi} \\
& \quad \leq\|u\|_{\infty}\left\|f_{T}\right\|_{\infty}\|f\|_{\varphi}+\sum_{i=1}^{n}\left\|\frac{\partial u}{\partial x_{i}}(f \circ T)\right\|_{\varphi}+\sum_{i=1}^{n}\left\|u \sum_{k=1}^{n}\left(\frac{\partial f}{\partial x_{k}} \circ T\right) \frac{\partial T_{k}}{\partial x_{i}}\right\|_{\varphi} \\
& \quad \leq\|u\|_{\infty}\left\|f_{T}\right\|_{\infty}\|f\|_{1, \varphi}+M_{1}\left\|f_{T}\right\|_{\varphi}\|f\|_{1, \varphi}+n M\|u\|_{\infty}\left\|f_{T}\right\|_{\infty}\|f\|_{1, \varphi} .
\end{aligned}
$$

Thus $\left\|\left(u C_{T}\right) f\right\|_{1, \varphi} \leq K\|f\|_{1 . \varphi}$ for some $K>0$.

We now give some additional conditions on $\Omega, u, T$ and $\varphi$ to obtain sufficient conditions for $u C_{T}$ to be compact.

THEOREM 3.2. With all the conditions stated in Lemma 3.1, let $\Omega \subset \mathbf{R}^{n}(n \geq 2)$ be an open set having the cone property ([1, Definition 4.3 Page 66]) with $\mu(\Omega)<\infty$. Let $u, \partial u / \partial x_{i}$ be continuous, $u T^{\prime}=0$ and $\left(\partial u / \partial x_{i}\right) T^{\prime}=0, i=1,2, \ldots, n$, where $T^{\prime}$ denotes the $n^{\text {th }}$ order Jacobian matrix of the first order classical partial derivatives $\partial T_{k} / \partial x_{i}$. If the Young function $\varphi$ also satisfies $\int_{1}^{\infty}\left(\varphi^{-1}(t) / t^{1+1 / n}\right) d t<\infty$, where $\varphi^{-1}(t)=\inf \{s>0: \varphi(s)>t\}$ is the right continuous inverse of $\varphi$, then the weighted composition operator $u C_{T}: f \mapsto u \cdot(f \circ T)$ is compact on the Orlicz-Sobolev space $W^{1 . \varphi}(\Omega)$.

PROOF. Let $\left\langle f_{m}\right\rangle$ be a sequence in $W^{1 . \varphi}(\Omega)$ with $\left\|f_{m}\right\|_{1 . \varphi} \leq 1$. We prove that there exists an element $g \in W^{1 . \varphi}(\Omega)$ and a subsequence $\left\langle f_{m_{k}}\right\rangle$ with $\left(u C_{T}\right)\left(f_{m_{k}}\right) \rightarrow g$ in $W^{1 . \varphi}(\Omega)$ as $k \rightarrow \infty$. Equivalently, it suffices to show that $u C_{T}\left(f_{m_{k}}\right) \rightarrow g$ in $L^{\varphi}(\Omega)$ and $\partial\left(u C_{T} f_{m_{k}}\right) / \partial x_{i}$ is bounded in $L^{\varphi}(\Omega)$. Let

$$
E=\bigcup_{i=0}^{n}\left\{x \in \Omega: \frac{\partial u(x)}{\partial x_{i}} \neq 0\right\}, \quad \frac{\partial u(x)}{\partial x_{0}} \equiv u .
$$

Then, since $u$ and $\partial u / \partial x_{i}$ are continuous, $E$ becomes an open subset of $\Omega$. Let $E=\bigcup_{i=1}^{\infty} \Omega_{i}$ where the $\Omega_{i} \mathrm{~s}$ are closed cubes with disjoint interiors in $\Omega$ [8, Theorem 1.11, Page 8]. Thus for all $x$ in $\Omega_{i}$ we have $T(x)=C_{i}$ for some $C_{i} \in \mathbf{R}^{n}$.

Now, from [1, Theorem 8.35, Page 252] it follows that $W^{1 . \varphi}(\Omega)$ can be embedded in $C(\Omega) \cap L^{\infty}(\Omega)$. Therefore we can consider the sequence $\left\langle f_{m}\right\rangle$ in $W^{1, \varphi}(\Omega)$ as a bounded sequence of continuous functions on $\Omega$. For $x \in \Omega_{1}$, we have

$$
\left\langle\left(f_{m} \circ T\right)(x)\right\rangle=\left\langle f_{m}(T(x))\right\rangle=\left\langle f_{m}\left(C_{1}\right)\right\rangle
$$


is a bounded sequence of real numbers and so there exists a subsequence $\left\langle f_{1, m}\right\rangle$ of $\left\langle f_{m}\right\rangle$ and $A_{1} \in \mathbf{R}$ such that

$$
f_{1, m}\left(C_{1}\right) \rightarrow A_{1}
$$

Similarly, for $x$ in $\Omega_{2}$, we can find a subsequence $\left\langle f_{2, m}\right\rangle$ of $\left\langle f_{1, m}\right\rangle$ and $A_{2} \in \mathbf{R}$ such that

$$
f_{2, m}\left(C_{2}\right) \quad \rightarrow \quad A_{2}
$$

Continuing in this way, by induction we obtain for each positive integer $i$, a real number $A_{i}$ and a subsequence $\left\langle f_{i, m}\right\rangle$ of $\left\langle f_{i-1, m}\right\rangle$ with

$$
f_{i, m}\left(C_{i}\right) \quad \rightarrow \quad A_{i} \quad \text { as } m \rightarrow \infty .
$$

For each positive integer $k$, take $f_{m_{k}}=f_{k, k}$. Thus by the above construction we obtain that for each $i$

$$
f_{m_{k}}\left(C_{i}\right) \quad \rightarrow \quad A_{i} \quad \text { as } k \rightarrow \infty .
$$

Therefore for $\epsilon>0$ we have, for sufficiently large $m_{k}$,

Let

$$
\left|f_{m_{k}}\left(C_{i}\right)-A_{i}\right|<\frac{\epsilon}{2^{i}} .
$$

$$
g(x)= \begin{cases}A_{i} u(x) & \text { if } x \in \Omega_{i} \\ 0 & \text { if } x \notin E=\bigcup_{i=1}^{\infty} \Omega_{i} .\end{cases}
$$

This means that if $u$ and $\partial u / \partial x_{i}(i=1,2, \ldots, n)$ do not vanish then we put

$$
g(x)=A_{i} u(x),
$$

while $g(x)=0$ if $u(x)=0=\partial u / \partial x_{i}$ for $i=1,2, \ldots, n$. Thus we have $g \in L^{\varphi}(\Omega)$.

We now show that $u \cdot\left(f_{m_{k}} \circ T\right) \rightarrow g$ in $L^{\varphi}(\Omega)$ as $k \rightarrow \infty$. For sufficiently large $m_{k}$, consider

$$
\begin{aligned}
\int_{\Omega} \varphi\left(\frac{\left|u \cdot\left(f_{m_{k}} \circ T\right)-g\right|}{\epsilon\|u\|_{\infty}\left[\varphi^{-1}\left(\frac{1}{\mu(E)}\right)\right]^{-1}}\right) d \mu & =\int_{E} \varphi\left(\frac{\left|u(x) f_{m_{k}}(T(x))-g(x)\right|}{\epsilon\|u\|_{\infty}\left[\varphi^{-1}\left(\frac{1}{\mu(E)}\right)\right]^{-1}}\right) d \mu \\
& =\sum_{i=1}^{\infty} \int_{\Omega_{i}} \varphi\left(\frac{|u(x)|\left|f_{m_{k}}\left(C_{i}\right)-A_{i}\right|}{\epsilon\|u\|_{\infty}\left[\varphi^{-1}\left(\frac{1}{\mu(E)}\right)\right]^{-1}}\right) d \mu \\
& \leq \sum_{i=1}^{\infty} \int_{\Omega_{i}} \varphi\left(\frac{1}{2^{i}}\left(\varphi^{-1}\left(\frac{1}{\mu(E)}\right)\right) d \mu\right. \\
& \leq \sum_{i=1}^{\infty} \frac{1}{2^{i}} \int_{\Omega_{i}} \varphi\left(\varphi^{-1}\left(\frac{1}{\mu(E)}\right)\right) d \mu \\
& \leq 1
\end{aligned}
$$


Therefore, by the definition of infimum, we have

$$
\left\|u \cdot\left(f_{m_{k}} \circ T\right)-g\right\|_{\varphi} \leq \epsilon\|u\|_{\infty}\left[\varphi^{-1}\left(\frac{1}{\mu(E)}\right)\right]^{-1}
$$

Hence $\left(u C_{T}\right)\left(f_{m_{k}}\right) \rightarrow g$ in $L^{\varphi}(\Omega)$ as $k \rightarrow \infty$.

Now $\left\|f_{m_{k}}\right\|_{1, \varphi} \leq 1$, therefore, by using Lemma 3.1, we have

$$
\left\|\frac{\partial}{\partial x_{i}}\left(u \cdot\left(f_{m_{k}} \circ T\right)\right)\right\|_{\varphi} \leq\left\|\frac{\partial}{\partial x_{i}}\left(u \cdot\left(f_{m_{k}} \circ T\right)\right)\right\|_{1 . \varphi} \leq K\left\|f_{m_{k}}\right\|_{1 . \varphi} \leq K .
$$

The result follows.

\section{References}

[1] Robert A. Adams. Sobolev Spaces (Academic Press. New York. 1975).

[2] S. C. Arora and M. Mukherjee, 'Compact composition operators on Sobolev spaces'. Indian J. Math. 37 (1995). 207-219.

[3] Y. Cui. H. Hudzik. Romesh Kumar and L. Maligranda. 'Composition operators in Orlicz spaces', J. Aust. Math. Soc. 76 (2004). 189-206.

[4] Herbert Kamowitz and Dennis Wortman. 'Compact weighted composition operators on Sobolev related spaces', Rocky Mountain J. Math. 17 (1987). 767-782.

[5] B. S. Komal and Shally Gupta. 'Composition operators on Orlicz spaces'. Indian J. Pure Appl. Math. 32 (2001). 1117-1122.

[6] A. Kufner. O. John and S. Fucik. Function Spaces (Noordhoff International Publishing, Leyden. 1977).

[7] Romesh Kumar. 'Composition operators on Orlicz spaces'. Integral Equations Operator Theory 29 (1997). 17-22.

[8] Richard L. Wheeden and Antoni Zygmund. Measure and Integral (Marcel Dekker Inc.. New York. 1977).

Department of Mathematics

Department of Mathematics

University of Delhi

PGDAV College

Delhi-1 10007

University of Delhi

India

Delhi-110065

e-mail: scarora@maths.du.ac.in

India

e-mail: gopaldatt@maths.du.ac.in

Department of Mathematics

SGTB Khalsa College

University of Delhi

Delhi-110007

India

e-mail: vermas@maths.du.ac.in 